\title{
Conventional povidone-iodine paint only technique vs. spray technique in preoperative antisepsis of anterior abdominal wall: A comparison of effectiveness and cost
}

\author{
K A S U A Kodithuwakku ${ }^{a}$, D M S T Gnanarathne ${ }^{b}$, S Hemapriya ${ }^{c}$, A C M Musthaq ${ }^{d}$
}

\begin{abstract}
Objectives

To assess the effectiveness of spray technique (aqueous based solution in a reusable sprayer) and conventional paint only technique in view of reducing abdominal wall bacteria and to assess the volume of povidone-iodine used in each technique and evaluate which technique is more cost-effective.

\section{Methodology}

The sample included 70 patients, who had elective Gynaecological surgeries at Teaching Hospital, Kandy (2014-2015). This was a non-randomized clinical trial. In all patients, half the abdomen was prepared using the conventional technique and the other half using the new spray technique. Prepreparation and post-preparation skin swabs were taken and cultured to assess the mixed bacterial colony counts. Two output variables were measured; percentage reduction of mixed bacterial colony counts between preoperative and postoperative cultures for the two methods separately and the volume of povidone-iodine used in each method.
\end{abstract}

\section{Results}

The mean percentage reductions in mixed bacterial colony count in spray method: $98.78 \%$ and in the conventional paint method: $98.74 \%$. $P$ value for the two sample t-test was 0.963 . Thus there is no significant difference between the mean percentage reductions in the mixed bacterial colony count in the two methods. The mean volume of povidone-iodine used in the spray method was $33.514 \mathrm{ml}$ with a corresponding $P$ value of 0.000 . Therefore the volume of povidone-iodine used in the spray method is significantly less than $50 \mathrm{ml}$ that is used in the conventional method. (corresponding 95\% upper bound for the $\mathrm{Cl}$ : 34.714).

\section{Conclusion}

Both the conventional paint only technique and the new spray technique are equally effective in reducing abdominal wall bacteria. The volume of povidoneiodine used in the spray method is significantly less than that of the conventional method.

Key words: Preoperative antisepsis, povidone-iodine, anterior abdominal wall preparation, spray method, paint method

Sri Lanka Journal of Obstetrics and Gynaecology

2018; 40: 85-91

DOI: http://doi.org/10.4038/sljog.v40i4.7870

a Senior Obstetrics and Gynaecology Registrar, WH Sunshine Hospital, Melbourne, Australia

${ }^{\mathrm{b}}$ Consultant Obstetrician and Gynaecologist, Base Hospital, Dickoya, Sri Lanka

c Consultant Obstetrician and Gynaecologist, Teaching Hospital, Kandy, Sri Lanka

d Acting Consultant Obstetrician and Gynaecologist, DGH Mulativ, Sri Lanka

Correspondence: KKASUA, e-mail: <sajithkodithuwakku@gmail.com>

Received 5 November 2018 and revised version accepted 10 December 2018.

http://orcid.org/0000-0003-4063-6786

Competing interest: The authors report no conflict of interest

This is an open-access article distributed under the terms of the Creative Commons Attribution 4.0 International License, which permits unrestricted use, distribution and reproduction in any medium provided the original author and source are credited. 


\section{Introduction}

Up to $15 \%$ of patients who undergo elective clean surgeries and $30 \%$ of patients who receive contaminated surgeries are estimated to develop postoperative wound infections. In the case of elective surgeries, these infections are mainly due to microorganisms in the skin surface ${ }^{1,2}$.

There are various antiseptics used for preoperative preparation of surgical sites. The most frequently used antiseptics include chlorhexidine and povidone-iodine, with aqueous media or alcohol used as the base. povidone-iodine is a stable chemical complex of polyvinylpyrrolidone and elemental iodine. It contains from $9.0 \%$ to $12.0 \%$ available iodine, calculated on a dry basis. Human clinical trials have shown the product to be superior to other iodine formulations in preoperative preparation of surgical sites ${ }^{3,4}$.

Commonly used cleaning techniques include spraying, scrub-paint and paint-only techniques. Studies have shown that spraying and scrub-paint techniques are equally effective in reducing abdominal wall bacteria when used preoperatively and allowed to air-dry for 3 minutes after application ${ }^{5}$. These studies used an alcohol-based povidone-iodine solution for the spraying technique, in accordance with the current recommendations. Additional studies have compared the effectiveness of the scrub-paint technique and the paint-only technique using an aqueous-based povidoneiodine solution ${ }^{6}$. However, no studies have assessed the effectiveness of the spraying technique versus the paint-only technique, which is commonly used in gynaecological practice.

The paint-only technique is most commonly used for preoperative skin preparation in gynaecological surgery in Sri Lanka; the practice is to clean the abdomen with 3 povidone-iodine-soaked gauze swabs on sponge holders. As this is not the exact method described in the standard guidelines, it will be referred to as the conventional paint-only method hereafter. In this technique, the povidone-iodine solution is poured into a 50ml Gully pot, where the cleaning swabs are then soaked. The remaining volume of the povidone-iodine solution in the Gully pot is discarded at the end of the surgery, as well as any solution soaked into gauze swabs not used for cleaning, which a waste of resources.
At the time of designing the study, a $500 \mathrm{ml}$ bottle of the aqueous-based povidone-iodine used in the study setting (manufactured by Medicom Superdrug Manufacturing) costs around Rs. 1200/-. The cost for a single-use $75 \mathrm{ml}$ alcohol-based povidone-iodine sprayer ranges from Rs 2200/- to 2500/-. Therefore, using the commercial sprayer is more expensive compared to the current paint-only technique. In this study, the same aqueous-based solution used in the paint-only technique will be used in a low-cost, commercially available, reusable hard plastic trigger sprayer for spraying.

Provided that the paint-only and spraying techniques are equally effective in reducing abdominal wall bacteria and the sterility of the sprayer is maintained between the surgeries and can be used for multiple surgeries, it is reasonable to hypothesise that cleaning can be done with a lesser volume of solution with the sprayer, thus making it more cost-effective.

\section{Methods}

The methods were pre-run to assess the baseline volumes of povidone-iodine solution used in each technique (spraying and paint-only). To assess the volume of povidone-iodine expelled from the sprayer with each puff, 20 full-stroke puffs were sprayed into a standard measuring cup and an average measurement was taken (volume/20, which is $1 \mathrm{ml}$ ). The sprayer nozzle was adjusted and fixed and was not altered throughout the study. For the paint technique, $50 \mathrm{ml}$ of povidone-iodine solution measured with a commercially available standard measuring cup was used for each patient.

The study was conducted on women who underwent elective gynaecological surgeries that involved a suprapubic transverse incision. Patients were recruited during their preoperative visit to the gynaecology clinic. Patients who had surgery for cancer or had comorbidities that increased their postoperative infection rates, such as diabetes and immunosuppressive states, were excluded from the study. Prior to their enrolment in the study, the patients were counselled and provided with written information regarding the research. If they agreed to participate, signed written consent was obtained.

The patients who consented to participate in the study were admitted 2 days prior to the surgery. On the 
morning of the surgery, hair was removed from the surgical site using a disposable razor blade. The patients were asked to bathe with non-medicated soap and water and given freshly laundered clothes before entering the operating room.

After anaesthesia was administered, a culture swab was rolled 3 times over the same line of skin $2 \mathrm{~cm}$ below the umbilicus in the midline to obtain a control mixed bacterial colony count. The swabs were immediately transferred to the microbiology laboratory and inoculated onto a human blood agar plate. Then half of the abdomen, as separated by a vertical line along the umbilicus, was prepared using the paint method, and the other half was prepared using the spray method. The right side was consistently cleaned with the spray, and the left side with the paint. The area cleaned by each method was from the xiphisternum down to the mid-thigh. Cleaning with the spray was done first, and the other half of the abdomen was covered using a general surgical towel.

In the paint-only technique, the specified area was prepared with povidone-iodine by the surgeon using three povidone-iodine soaked gauze swabs on sponge holders. The cleaning process was started at the incision site from medial to lateral, painted with povidone-iodine with one stroke on each skin area. The process was repeated thrice using different povidone-iodine soaked gauze swabs.

Umbilicus was always cleaned using the paint technique. Other half of the abdomen was then prepared with the spray technique by the surgeon. Spraying was done until the specified area is satisfactorily covered with a layer solution. The sprayer was triggered to deliver a full stroke each time and the number of puffs used was counted and recorded for each patient. The volume used for the preparation was calculated by multiplying the number of puffs by two, as for the whole abdomen, into the pre-calculated average volume of a puff (Number of puffs $\times 2 \times 1 \mathrm{ml}=$ Volume used for preparation).

The sprayer was kept on a small trolley covered with a sterile surgical towel and it was used by the surgeon with sterile gloves before touching the patient after scrubbing up.
After using, the sprayer was placed back on the trolley and taken back to the sterile corner in the operating theatre by an assistant.

Once the sprayer went out of solution, it was cleaned and sterilized using liquid type peracetic acid solution (peracetic acid $0.13 \%$, hydrogen peroxide $3.15 \%$, isopropanol 1.9\%) and refilled. Two similar sprayers were used alternatively in the study.

Two swab cultures, one from each side of the abdomen were taken with three back and forth rolling movements of the swab on a three-inch vertical line, midway between the umbilicus and the anterior superior iliac spine after 3 minutes after application of povidoneiodine. The swabs were immediately transferred to the Microbiology lab for inoculation.

The pre-preparation and post-preparation culture plates were incubated at $37^{\circ} \mathrm{C}$ for 48 hours, and the mixed bacterial colony count was taken and recorded.

The percentage reduction in mixed bacterial colony counts was calculated by subtracting the colony count after preparation from the control colony count, divided by the control colony account, and then multiplied by 100 .

\section{Results}

For the analysis of data for the effectiveness in reducing abdominal wall bacteria, a two sample t-test was used. The average for colony count reduction in each arm was calculated and compared to see if there is a significant difference between the two techniques. A $P$ value of 0.05 was considered statistically significant in the analysis. The cultures with zero growth with each method were taken as a100\% reduction.

To assess the statistical significance of the difference in volumes of the solution used in each technique a one sample t- test was used. The average volume used in the spray technique was compared with $50 \mathrm{ml}$, which was the average volume for the paint only technique. AP value of 0.05 was considered statistically significant in the analysis.

Analyses were performed with Minitab 17 statistical software (Updated March, 2015). 
Table 1. Results of two sample t-test on average percentage reductions of mixed microbial colony count in spray method and conventional paint method

\begin{tabular}{|lccccc|}
\hline \multicolumn{5}{|c|}{$\begin{array}{c}\text { Two-sample T for spray method vs } \\
\text { conventional method }\end{array}$} \\
\hline & $\mathrm{N}$ & Mean & St Dev & SE Mean \\
\hline $\begin{array}{l}\text { Spray method } \\
\begin{array}{l}\text { Conventional } \\
\text { method }\end{array}\end{array}$ & 70 & 0.9878 & 0.0485 & 0.0058 \\
\hline
\end{tabular}

Difference $=\mu$ (Spray method $)-\mu$ (Conventional method $)$

Estimate for difference: 0.00039

95\% CI for difference: (-0.01596, 0.01673)

$\mathrm{T}$-Test of difference $=0(\mathrm{vs} \neq)$ : T-Value $=0.05$

$\mathrm{P}$-Value $=0.963 \mathrm{DF}=137$
Two-sample t-test was used to assess if there is a significant difference between the mean percentage reductions in mixed bacterial colony counts in the spray method and the conventional paint method. $\mu$ (Spray Method $)=\mu$ (Conventional Method) was taken as the null hypothesis and $\mu$ (Spray Method) $\neq \mu$ (Conventional Method) as the alternative hypothesis. The $\mathrm{P}$ value for the test was 0.963 . Therefore the null hypothesis is not rejected. Thus there is no significant difference between the mean percentage reductions in the mixed bacterial colony count in the two methods. Therefore a conclusion can be drawn that both the cleaning methods are equally effective at preoperative antisepsis of the anterior abdominal wall.

Chart 1. Histogram of percentage reduction of mixed microbial colony counts in spray method vs. conventional paint method

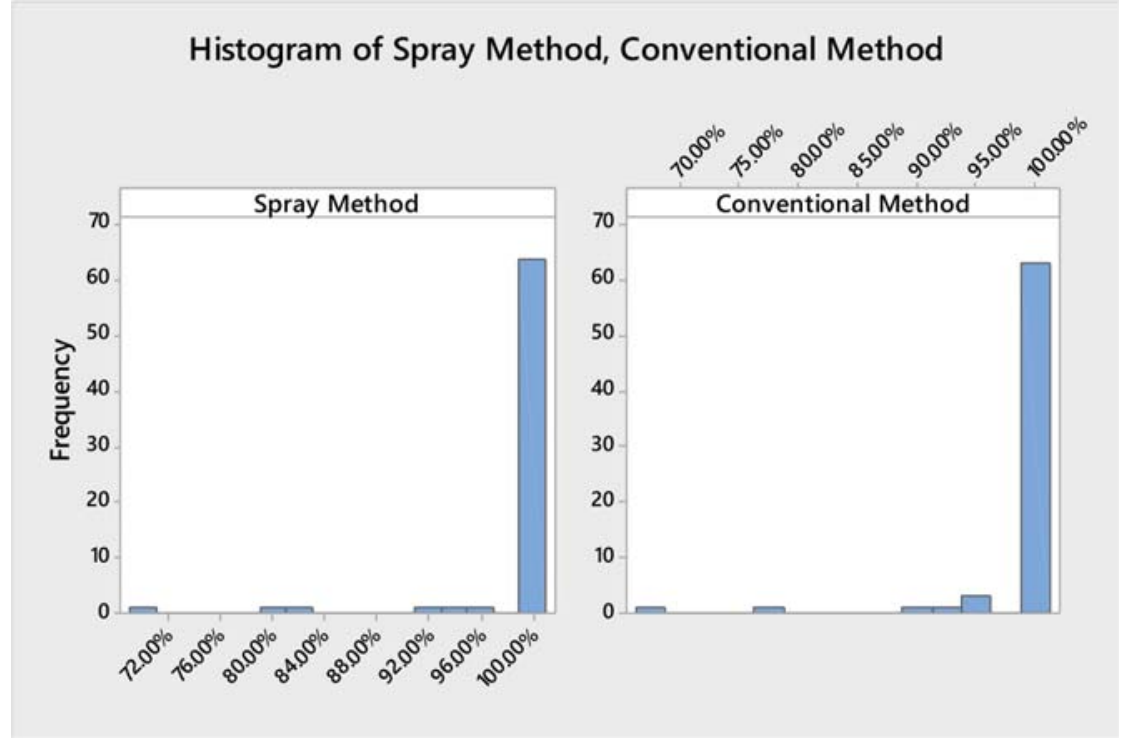

Histogram showing the percentage reductions of mixed bacterial colony count in spray method (left) and the conventional paint method (right).

Table 2. Results of one sample t-test on average volumes of povidone-iodine used in spray method and conventional paint method

\begin{tabular}{|lcccccccc|}
\hline \multicolumn{2}{|c|}{ Test of $\boldsymbol{\mu}=\mathbf{5 0}$ vs $<\mathbf{5 0}$} \\
\hline Variable & $\mathrm{N}$ & Mean & StDev & SE Mean & $95 \%$ Upper Bound & $\mathrm{T}$ & $\mathrm{P}$ \\
\hline Volume & 70 & 33.514 & 6.019 & 0.719 & 34.714 & -22.92 & 0.000 \\
\hline
\end{tabular}


The mean volume of povidone-iodine used in the conventional method was fixed and it was $50 \mathrm{ml}$ for all the patients. The mean volume of povidone-iodine used in the spray method was $33.514 \mathrm{ml}$. One sample T-test was used to see if the difference is significant between the mean volumes of povidone-iodine used in the two methods. $\mu=50$ was taken as the null hypothesis and $\mu<50$ as the alternative hypothesis. The corresponding $\mathrm{P}$ value for the test came as 0.000 , which implies that the null hypothesis should be rejected. Thus it could be concluded that the volume of povidone-iodine used in the spray method is significantly less than that is used in the conventional method with a corresponding 95\% upper bound for the CI: 34.714 .

\section{Chart 2. Histogram of frequency vs. volume of povidone-iodine used in the spray method}

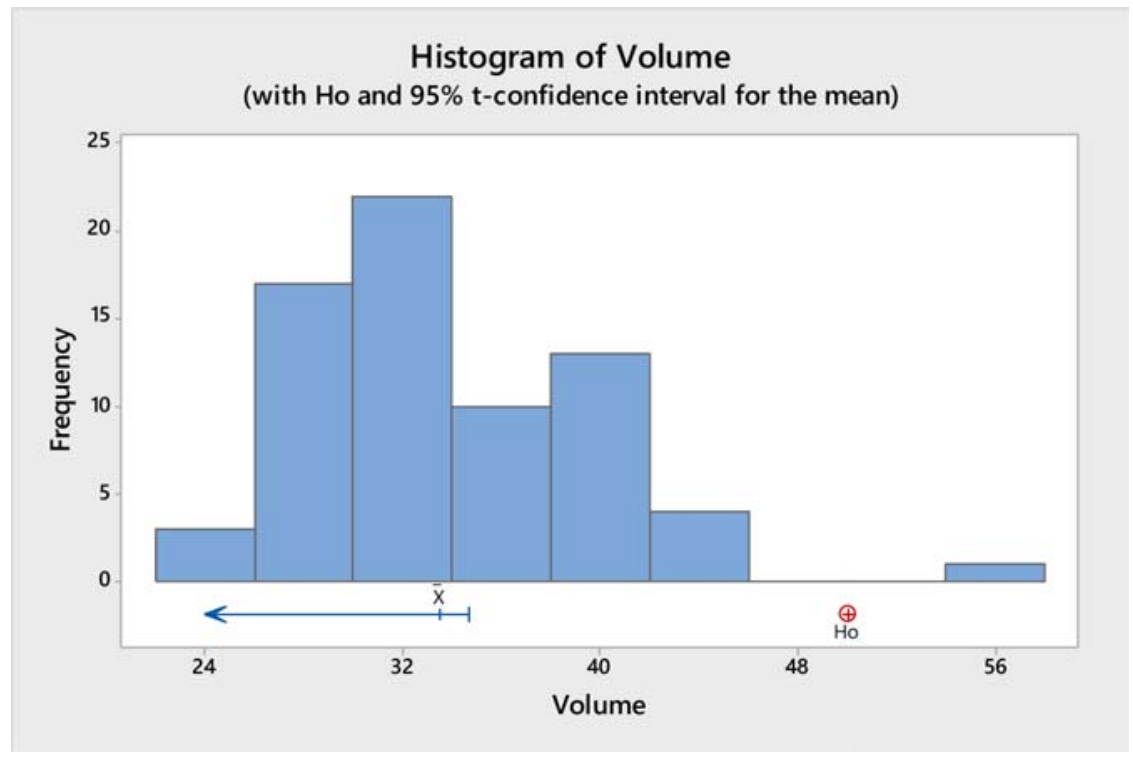

The volumes used in the spray method are apparently less than $50 \mathrm{ml}$ which is the fixed volume of povidoneiodine used in the conventional paint only method.

The volume used in one of the subjects in the spray group was $56 \mathrm{ml}$, which was more than the average volume used in the conventional method (50ml). Since this is just one value, it could be regarded as an outlier, which does not have an effect on the final outcome of the study.

\section{Discussion}

There are several different antiseptic solutions and preoperative skin preparation techniques described in the literature. These antiseptic solutions include povidone-iodine, chlorhexidine, and alcohol, and the techniques include the paint-only, scrub-and-paint, and spray methods. In Sri Lanka, the most common technique used in gynaecological practice is the paintonly method with an aqueous-based povidone-iodine solution. The exact technique used in the study was described in detail in the introduction and referred to as the conventional paint-only technique.

The objective of this study was not to look for a better antiseptic method but to see if the new spray method can be used as an equally effective but cheaper alternative to the conventional method.

The study design was a non-randomized clinical trial. Randomization was not done as each subject comes under both arms of the study. The sample size was 70 , and the age distribution of the study group was between 23 to 72 years old, excluding patients with cancer and those with co-morbidities that pre-disposed them to infections. Two output variables were measured: the percentage reduction in bacteria between the pre-operative and post-operative cultures for the two methods separately and the volume of povidoneiodine used in each method. Each variable was measured separately for each subject, and the t-test 
was used to assess any significant difference between the average values. A 95\% confidence level was accepted for statistical significance.

The mean percentage reduction in the mixed bacterial colony count in each method was as follows: the spray method was $98.78 \%$, and the conventional paint-only method was $98.74 \%$. The p-value for the test was 0.963 . Thus, there is no significant difference between the mean percentages of reduction in the mixed bacterial colony counts in the two methods, so we concluded that the cleaning methods are equally effective.

The mean volume of povidone-iodine used in the conventional method was $50 \mathrm{ml}$ and for the spray method it was $33.514 \mathrm{ml}$, with a corresponding p-value of 0.000 . Therefore, the volume of povidone-iodine used in the spray method is significantly less than that used in the conventional method (corresponding 95\% upper bound for the CI: 34.714). The volume used on one of the subjects in the spray group was $56 \mathrm{ml}$, which was more than the average volume used in the conventional method $(50 \mathrm{ml})$. Since this is just one value, it maybe regarded as an outlier and does not have any effect on the final outcome of the study.

This study concludes that both the conventional and new techniques are equally effective in reducing abdominal wall bacteria, and the new method uses less antiseptic solution. Therefore, the new method is more cost effective and can be used as an alternative to the conventional method.

Sri Lanka is a developing country that provides free health care services to the entire population. A considerable amount of the health budget is spent on surgical patients and the various aspects of surgery, and preoperative skin antisepsis is one major component of these costs. In terms of reducing health costs, it is high time to look into cheaper and more effective antiseptic techniques.

On average, the gynaecology unit in the study setting accommodates 20 abdominal surgeries a week, which amounts to 1,040 surgeries a year. The yearly cost for the antiseptic solution with the conventional method would be Rs. 124,800 $(1040 \times 0.051 \times$ Rs. 2400$)$, and with the new spray method, this cost would be Rs. 93,216 $(1040 \times 0.03351 \times$ Rs. 2400), for a saving of Rs. 31,584 each year. If the method can be introduced into other surgical specialties even in just one hospital in the country, this saving could be substantial. One of the main obstacles for further development of country's health sector towards providing a better patient care is the limitation in the budget. Savings made by cheap yet effective alternative methods as suggested in this study can be utilised in much needed areas of health, such as disease prevention, health education, continuous professional development, patient safety and equipment.

Using an aqueous-based solution in sprayers was not recommended under current guidelines as it does not show an adequate level of sterilization for surgery compared to the paint-only and scrub-and-paint techniques. On the other hand, the conventional method of abdominal wall preparation used in the study was different from that described in the standard guidelines (Best Practice Guidelines [Winnipeg Regional Health Authority (WRHA) Canada] by the surgeon, 2011), and it has been described in the introduction and methodology as the conventional method.

According to our observations, in the conventional method, a more or less fixed volume of povidone-iodine is used for all patients; most of the time, any remaining solution is wasted. The volume of povidone-iodine that was discarded in the conventional method was not objectively measured in the study. Salvaging this discarded volume of the solution seemed difficult for a couple of reasons. First, taking a lesser volume of the antiseptic solution in the Gully pot to begin with is a challenge as the volume of the solution needed for pre-operative skin preparation depends on the patient's build and body surface area, which differs from one patient to the other. Secondly, as the painting the abdominal wall is done with gauze swabs in a sponge holder, and the swabs absorb only a limited amount of solution at a time and therefore, in most cases, it is necessary to dip the gauze swabs back into the solution to complete the process, which contaminates the solution in the Gully pot and makes it unusable on another patient. Using a sprayer addresses these issues and is a good alternative to the conventional cleaning method.

The surgeons' satisfaction regarding the use of a sprayer as opposed to other techniques was not objectively assessed during this study. However, in casual discussions with the surgeons, most of them liked the sprayer due to its convenience. This obser- 
vation should be objectively studied further before drawing any conclusions. Another advantage observed in the study was that the sprayer does not require any of the sterile equipment needed for the paint method (sponge holders, gully pots, gauze swabs, etc.). This may be added to the cost effectiveness of the spray method, which again needs further objective assessment.

\section{Conclusions and recommendations}

The two methods compared in the study; conventional paint only method and new spray method, are equally effective in reducing the bacterial colony counts and therefore in preoperative preparation of the anterior abdominal wall. Thus aqueous based povidone-iodine solution can be used in a reusable sprayer with comparable antiseptic effectiveness to that of the alcohol-based solution.

The volume of povidone-iodine used in the spray method is significantly less than that is used in the conventional paint only method. Therefore it can be concluded that using aqueous based povidone-iodine solution in a reusable sprayer is cost-effective.

Further studies should be carried out to compare the spray method with other standard cleaning methods using larger samples and taking into account the confounding factors to find out the success of the new method.

\section{Acknowledgements}

We would like to thank Mr. Kasun Rathnayake for his assistance in the statistical analysis of the results of the study and Dr. S Lanerolle and Dr. P K Gunathilake for their support.

\section{Authors contributions}

All authors contributed equally to the study.

\section{References}

1. Dumville JC, McFarlane E, Edwards P, Lipp A, Holmes A, Liu Z. Preoperative skin antiseptics for preventing surgical wound infections after clean surgery. Cochrane Database of Systematic Reviews. 2015 (4). Pub Med PMID: CD003949.

2. Vagholkar K, Julka K. Preoperative Skin Preparation: Which Is The Best Method? The Internet Journal of Surgery. 2012; 28(4).

3. Sneader W. Chemical Medicines. Drug Discovery: John Wiley \& Sons, Ltd; 2006. p. 41-73.

4. Darouiche RO, Wall MJJ, Itani KMF, Otterson MF, Webb AL, Carrick MM, et al. ChlorhexidineAlcohol versus Povidone-Iodine for Surgical-Site Antisepsis. N Engl J Med. 2010; 362(1):18-26. PubMed PMID: 20054046.

5. Moen MD, Noone MB, Kirson I. Povidone-iodine spray technique versus traditional scrub-paint technique for preoperative abdominal wall preparation. Am J Obstet Gynecol. 2002 12//; 7(6):1434-7.

6. Ellenhorn JDI, Smith DD, Schwarz RE, Kawachi MH, Wilson TG, McGonigle KF, et al. Paint-Only Is Equivalent to Scrub-and-Paint in Preoperative Preparation of Abdominal Surgery Sites. J Am Coll Surg. 2005 11//; 201(5): 737-41. 